\title{
The Modeling of the Young's Interference Experiment in terms of Single-photon wave function in the coordinate representation
}

\author{
Alexandr P. Davydov \\ Dept. of Applied and Theoretical Physics of Institute of \\ Natural Sciences and Standardization \\ Nosov Magnitogorsk State Technical University \\ Magnitogorsk, Russia \\ ap-dav@yandex.ru
}

\author{
Tatiana P. Zlydneva \\ Dept. of Applied Mathematics and Informatics of Institute \\ of Natural Sciences and Standardization \\ Nosov Magnitogorsk State Technical University \\ Magnitogorsk, Russia \\ tapazl@yandex.ru
}

\begin{abstract}
The main principles of photon quantum mechanics describing its one-particle states with the help of the wave function in coordinate representations are given. This wave function (packet) is the superposition of the basic bivectors that are generalized eigenfunctions of energy, momentum, and helicity operators. The quantum-mechanical approach is offered for an explanation of the interference Young's experiment. This explanation is especially important for new interpretation of the non-laser method of obtaining the interference by the amplitude division, as in this case it is traditionally assumed that the radiation of one light train of a single atom interferes with itself. In our explanation, not both "halves" of some real train ("scrap") of the electromagnetic wave interfere with each other, but the both terms of the photon's wave function do this. Nevertheless, the wave function is not directly measured by experience, and therefore it does not exist as a physical object. The attraction of the wave function to the explanation of single-photon interference obtained "by the division of the wave front" is obviously also necessary. Therefore, the situation for the photons emitted by individual atoms (and also, obviously, by laser), is absolutely similar to the situation with the particles having mass, whose distribution in space is described by wave function in coordinate representation. It is pointed out that in fact the photon is not some "formed" quantum particle, but it is a quasi-particle arising when a certain spin wave propagates in a physical vacuum at Planck distances.
\end{abstract}

Keywords-Schrödinger equation; Maxwell's equations; wave packet; probability density; bivector; detector; extreme maximon, friedmon, planckeon; Planck's parameters; wave-particle duality.

\section{INTRODUCTION}

At present, it is customary to assume that the wave-particle properties of particles having mass are described by a wave function in a coordinate representation satisfying, for example, the Schrödinger or Dirac equation. In relation to wave phenomena of light, such as its interference in Young's experiment, the wave approach of classical electrodynamics is still applied. In [1], on the basis of the illustration of results of modeling in [2-6] of propagation in space-time of the photon wave packet corresponding to short-pulse laser radiation, a general idea was suggested for explaining of emergence of the interference with single photons in Young's double-slit experiment, using the photon wave function in coordinate representation. The purpose of this article is to take the next step in this direction. Namely, by choosing a certain form of complex polarization vectors and modeling the momentum distribution in the single-particle state of the photon, so that its wave function in the coordinate representation described the spherical diverging wave emanating from one (almost point) hole, we will further show that in the corresponding geometry, usually used in classical electrodynamics to explain Young's experiment, the results are exactly the same as in classical electrodynamics. Concretely, interference is determined by the usual phase difference of two spherical waves emitted by two (almost point) holes of this experiment. Thus, it can be concluded that the incorrect explanation of Young's experiment by classical electrodynamics (based on the interference of different "halves" of the same train of an electromagnetic wave emitted from each atom), nevertheless, gives exactly the same result as its correct explanation with the help of the photon wave function in the coordinate representation. However, from our point of view, it is also necessary to clarify what is understood by the photon itself. In the elementary particle physics (including, in the so-called standard model), the photon is considered as one of the fundamental point particles. In our opinion, the photon is not a particle but it is the quasiparticle, because its propagation in space is a consequence of the propagation of some excited quantum state of the physical vacuum. The excitation of a certain spin wave in the vacuum, similar to a magnon in a solid, is the most probable. However, the nature of the corresponding excitation can turn out to be quite more complicated; for example, the existence of vector dominance in the strong interaction of photons with hadrons indicates this.

\section{Photon Wave Function in Coordinate REPRESENTATION}

For a long time after the publication [7] it is customary to assume (See [8-14] for more details) that the photon wave function in coordinate space cannot be constructed although in momentum representation it is widely used, in particular in quantum electrodynamics. The reason for this is the zero rest mass photon, as it's well-known. Nevertheless, for the photon 
it is also possible to construct the wave function in the coordinate representation ("a wave packet"), if it is intended not to specify the photon localization probability, for example in the spirit of electron in atom, but to indicate the probability density of its detection in any space point, and at any time moment, in particular to identify the probability of its hit into "quite" dot detector (whose dimensions are much smaller than the area where the photon can be detected in principle). However, in the mid 90-ies of the last century, the works [1518] have begun to appear in which at interpretation of photon wave function the accent has been moved from the density of probability of photon localization to the density of probability of its detection in a some given spatial point, although the term "localization" sometimes continued to be used. New ideas at first were spreading with high inertness, since all optical phenomena had been well explained within classical or quantum electrodynamics. To overcoming of this inertness, even applied "metaphysical language" (see [19-24], etc.) didn't facilitate, with the use of the term "photon wave function", because the normalization on unit probability was not offered in case of the coordinate representation of this function. One of the fullest reviews differentiating both terminology, and sphere of applying of the photon wave function, is the review [25], written at a boundary of real designing and gradual introduction into the science of the photon wave function in coordinate representation. In it, as if summing up of all the research in the field of the optical phenomena, it was claimed that single-photon states can't almost be realized, and the photon in "metaphysical" language was represented as the certain object which has a little sense and is not amenable to experimental observation. The situation began to change with the emergence of the necessity for new experiments in purely methodological field of quantum mechanics (for example, when checking the Bell inequalities and quantum non-locality), and the practical applications of quantum optics (quantum cryptography, quantum teleportation, quantum computing). These experiments have led to the development of sources (see [26-29]) and detectors ([30-34]) of single photons.

Evidently, the photons are perceived in these experiments as carriers of elementary information units. Hence, the need for constructing the wave function of a photon in the coordinate representation becomes again urgent. In [35-45] and others the single-partial wave function of the photon got further development in theoretical substantiation.

In [2-6] for its evident illustration the modeling of free propagation in space of the wave packet describing the singlephoton state corresponding to laser radiation of duration $80 \mathrm{fs}$ with the central wavelength of 10 microns, with Gaussian distribution on the momentum (of photon) presented in this packet, is carried out. As a result of modeling, the character of an expansion of a wave packet is established: its spatial form from an initial "spherical" shape evolves into a certain "coneshaped", reminding a picture of Cerenkov radiation, since the peripheral parts of probability density of a packet lag behind the central part moving with speed of light $c$ in vacuum.

The description of the propagation of this wave packet completely obeys to quantum mechanics, and this packet in pure form represents the wave function of the photon in coordinate representation. Accordingly, the concept of group speed which is used in classical electrodynamics is initially not applicable to it. However, according to the quantummechanical principles, for this packet it is possible to calculate the average coordinate (radius vector) of detection of the photon at any given time, and hence the average velocity of the movement in space of this wave packet. It has turned out that the average velocity of this wave packet is considerably smaller than the speed of light $c$. This velocity is determined in fact by only one parameter, characterizing the momentum distribution of the photon in this state.

In $[46,47]$ a good agreement is found for the reduction of the center velocity of the wave packet simulated in [2-6] using the Gaussian momentum distribution within the framework of the constructed quantum mechanics of the photon (see $[6,18$, $38,43]$ ), with result of recent experiment [48], in which a "reduction" of the group velocity of light was established in Gaussian and Bessel beams, described by classical electrodynamics, but applied to individual photons. The explanation of this "reduction of the speed of light", given in $[46,47]$ within the framework of quantum mechanics of the photon, as a whole does not contradict the common ideas expressed in [49-50], etc., based on general concepts of classical electrodynamics and quantum mechanics.

According to $[6,38,43]$ the photon wave function in coordinate representation has the following form:

$$
\begin{aligned}
& \Psi^{( \pm)}(\mathbf{r}, t)=\int b(\mathbf{k}, \pm 1) \frac{\mathrm{e}_{ \pm 1}(\mathbf{k})}{(2 \pi)^{3 / 2}} e^{i(\mathbf{k r} \mu k c t)}\left(\begin{array}{l}
1 \\
0
\end{array}\right) d^{3} \mathbf{k}+ \\
& +\int[b(-\mathbf{k}, \mu 1)]^{*} \frac{\mathrm{e}_{\mu 1}(\mathbf{k})}{(2 \pi)^{3 / 2}} e^{i(\mathbf{k r} \mu k c t)}\left(\begin{array}{l}
0 \\
1
\end{array}\right) d^{3} \mathbf{k},
\end{aligned}
$$

where the top sign of all indexes corresponds to positive energy of photon, and the lower sign answers to negative energy, hypothetically possible; coefficients $b(\mathbf{k}, \lambda)$ are basically arbitrary, but if the photon state initially set by means of the electric (E) and magnetic $(\mathbf{H})$ fields intensities (in Gaussian System $), b(\mathbf{k}, \lambda)$ are expressed through them, as well as they satisfy to the normalization condition for the wave packet (1):

$$
\begin{gathered}
\left\langle\Psi^{( \pm)} \mid \Psi^{( \pm)}\right\rangle \equiv \int d^{3} \mathbf{r}\left[\Psi^{( \pm)}(\mathbf{r}, t)\right]^{+} \Psi^{( \pm)}(\mathbf{r}, t) \equiv \int d^{3} \mathbf{r} \rho_{P}^{( \pm)}(\mathbf{r}, t)= \\
=\int d^{3} \mathbf{k}\left[\Psi^{( \pm)}(\mathbf{k}, t)\right]^{+} \Psi^{( \pm)}(\mathbf{k}, t) \equiv \int d^{3} \mathbf{k} \rho_{P}^{( \pm)}(\mathbf{k})=1
\end{gathered}
$$

Here photon detection probability density in coordinate and momentum space (more precisely in the wave vector $\mathbf{k}=\mathbf{p} / \eta$ space, where $\mathbf{p}$ is photon momentum), respectively is equal to

$$
\begin{gathered}
\rho_{P}^{( \pm)}(\mathbf{r}, t)=\left[\Psi^{( \pm)}(\mathbf{r}, t)\right]^{+} \Psi^{( \pm)}(\mathbf{r}, t), \\
\rho_{P}^{( \pm)}(\mathbf{k}, t)=\left[\Psi^{( \pm)}(\mathbf{k}, t)\right]^{+} \Psi^{( \pm)}(\mathbf{k}, t)
\end{gathered}
$$


moreover, in accordance with (1) the photon wave function in momentum representation is equal to

$$
\begin{gathered}
\Psi^{( \pm)}(\mathbf{k}, t) \equiv \frac{1}{(2 \pi)^{3 / 2}} \int e^{-i \mathbf{k r}} \Psi^{( \pm)}(\mathbf{r}, t) d^{3} \mathbf{r}= \\
=e^{\mu i k c t}\left\{b(\mathbf{k}, \pm 1) e_{ \pm 1}(\mathbf{k})\left(\begin{array}{l}
1 \\
0
\end{array}\right)+[b(-\mathbf{k}, \mu 1)]^{*} e_{\mu 1}(\mathbf{k})\left(\begin{array}{l}
0 \\
1
\end{array}\right)\right\}
\end{gathered}
$$

Therefore photon detection probability density in the momentum space actually doesn't depend on time and it is

$$
\rho_{P}^{( \pm)}(\mathbf{k})=|b(\mathbf{k}, \pm 1)|^{2}+|b(-\mathbf{k}, \mu 1)|=\sum_{\lambda}|b(\mathbf{k}, \lambda)|^{2}
$$

where $\lambda= \pm 1$ takes two possible values of the photon helicity.

Equation (7) follows from (4) - (6), taking into account the properties of orthonormality of the complex polarization vectors

$$
\mathbf{e}_{\lambda}(\mathbf{k})=\left[\mathbf{e}_{I}(\mathbf{k})+i \lambda \mathbf{e}_{I I}(\mathbf{k})\right] / \sqrt{2}
$$

namely

$$
\left(\mathbf{e}_{\lambda^{\prime}}^{*} \mathbf{e}_{\lambda}\right)=\delta_{\lambda^{\prime} \lambda} ; \quad \mathrm{e}_{\lambda^{\prime}}^{+} \mathrm{e}_{\lambda}=\delta_{\lambda^{\prime} \lambda}
$$

In addition, with $\mathbf{n}=\mathbf{k} / k$ the following relations take place:

$$
\begin{gathered}
\mathbf{e}_{\lambda}(\mathbf{n})=\mathbf{e}_{-\lambda}(-\mathbf{n}) ; \quad\left[\mathbf{e}_{\lambda}(\mathbf{k})\right]^{*}=\mathbf{e}_{-\lambda}(\mathbf{k})=\mathbf{e}_{\lambda}(-\mathbf{k}), \\
\left|\mathbf{e}_{I}\right|=\left|\mathbf{e}_{I I}\right|=1 ; \quad\left(\mathbf{e}_{I} \mathbf{n}\right)=\left(\mathbf{e}_{I I} \mathbf{n}\right)=\left(\mathbf{e}_{I} \mathbf{e}_{I I}\right)=0, \\
\mathbf{e}_{I}(\mathbf{n})=\mathbf{e}_{I}(-\mathbf{n}) ; \quad \mathbf{e}_{I I}=\left\lfloor\mathbf{n} \times \mathbf{e}_{I}\right\rfloor, \\
\mathbf{n}=\left[\mathbf{e}_{I} \times \mathbf{e}_{I I}\right]=i \lambda\left[\mathbf{e}_{\lambda} \times \mathbf{e}_{\lambda}^{*}\right]=\lambda \mathrm{e}_{\lambda}^{+}(\mathbf{k}) \hat{\mathbf{s}} \mathrm{e}_{\lambda}(\mathbf{k}),
\end{gathered}
$$

where $\hat{\mathbf{s}}$ is the operator of the photon spin in vector representation:

$$
\begin{gathered}
\hat{\mathbf{s}}=\mathbf{e}_{x} \hat{\mathbf{s}}_{x}+\mathbf{e}_{y} \hat{\mathbf{s}}_{y}+\mathbf{e}_{z} \hat{\mathbf{s}}_{z}= \\
=\mathbf{e}_{x}\left(\begin{array}{ccc}
0 & 0 & 0 \\
0 & 0 & -i \\
0 & i & 0
\end{array}\right)+\mathbf{e}_{y}\left(\begin{array}{ccc}
0 & 0 & i \\
0 & 0 & 0 \\
-i & 0 & 0
\end{array}\right)+\mathbf{e}_{z}\left(\begin{array}{ccc}
0 & -i & 0 \\
i & 0 & 0 \\
0 & 0 & 0
\end{array}\right)=i\left(\begin{array}{ccc}
0 & -\mathbf{e}_{z} \mathbf{e}_{y} \\
\mathbf{e}_{z} & 0 & -\mathbf{e}_{x} \\
-\mathbf{e}_{y} & \mathbf{e}_{x} & 0
\end{array}\right)
\end{gathered}
$$

where $\mathbf{e}_{x}, \mathbf{e}_{y}, \mathbf{e}_{z}$ are the basis vectors of $x, y, z$ axes.

The photon wave function $\Psi^{( \pm)}(\mathbf{r}, t)$ in coordinate representation satisfies to the equation of the Schrödinger equation type

$$
i \eta \frac{\partial \Psi^{( \pm)}(\mathbf{r}, t)}{\partial t}=\hat{H}_{\mathrm{bv}} \Psi^{( \pm)}(\mathbf{r}, t),
$$

where

$$
\hat{H}_{\mathrm{bv}}=c\left(\hat{\boldsymbol{\alpha}}_{\mathrm{bv}} \hat{\mathbf{p}}\right)=\frac{c}{s}\left(\begin{array}{cc}
(\hat{\mathbf{s}} \hat{\mathbf{p}}) & 0 \\
0 & -(\hat{\mathbf{s}} \hat{\mathbf{p}})
\end{array}\right)
$$

is the Hamiltonian operator of free photon (having spin $s=1$ ) in bivector representation, in which its spin vector operator is equal to

$$
\hat{\mathbf{S}}=\mathbf{e}_{x} \hat{\mathrm{S}}_{x}+\mathbf{e}_{y} \hat{\mathrm{S}}_{y}+\mathbf{e}_{z} \hat{\mathrm{S}}_{z}=\left(\begin{array}{cc}
\hat{\mathbf{s}} & 0 \\
0 & \hat{\mathbf{s}}
\end{array}\right)
$$

$\hat{\mathbf{p}}=-i \eta \hat{\nabla}$ is particle momentum operator; the matrix $\hat{\alpha}_{\mathrm{bv}}$ in bivector representation has form

$$
\hat{\boldsymbol{\alpha}}_{\mathrm{bv}}=\left(\begin{array}{cc}
\hat{\mathbf{s}} & 0 \\
0 & -\hat{\mathbf{s}}
\end{array}\right)
$$

Equation (16) is similar to the equation, which is satisfied by bivector $\Phi_{\mathrm{bv}}=\left(\begin{array}{c}\xi \\ \eta\end{array}\right)$, with which it is also possible to describe [13] the photon state in the coordinate representation. Here the physical quantities $\xi$ and $\eta$ (in matrix form) are

$$
\xi=\left(\begin{array}{c}
\mathrm{E}_{x}+i \mathrm{H}_{x} \\
\mathrm{E}_{y}+i \mathrm{H}_{y} \\
\mathrm{E}_{z}+i \mathrm{H}_{z}
\end{array}\right) ; \quad \eta=\left(\begin{array}{c}
\mathrm{E}_{x}-i \mathrm{H}_{x} \\
\mathrm{E}_{y}-i \mathrm{H}_{y} \\
\mathrm{E}_{z}-i \mathrm{H}_{z}
\end{array}\right),
$$

but they are independent quantities from each other [13]. The bivector $\Phi_{b v}$ satisfies to the equation of type (16):

$$
i \eta \frac{\partial \Phi_{\mathrm{bv}}{ }^{( \pm)}(\mathbf{r}, t)}{\partial t}=\hat{H}_{\mathrm{bv}} \Phi_{\mathrm{bv}}{ }^{( \pm)}(\mathbf{r}, t),
$$

which is the consequence $[6,38,43]$ of the Maxwell equations written in the Majorana form [51, 13]

$i \eta \frac{\partial \xi}{\partial t}=c(\hat{\mathbf{s}} \hat{\mathbf{p}}) \xi ; i \eta \frac{\partial \eta}{\partial t}=-c(\hat{\mathbf{s}} \hat{\mathbf{p}}) \eta ;(\hat{\mathbf{p}} \xi)=0 ;(\hat{\mathbf{p}} \eta)=0$

Four independent solutions of (21) which are automatically satisfying to (22), and also being eigenfunctions of the helicity operator

$$
\hat{\Lambda}=\frac{(\hat{\mathbf{S}} \hat{\mathbf{p}})}{s p}=\frac{(\hat{\mathbf{S}} \hat{\mathbf{p}})}{p}=\frac{1}{p}\left(\begin{array}{cc}
(\hat{\mathbf{s}} \hat{\mathbf{p}}) & 0 \\
0 & (\hat{\mathbf{s}} \hat{\mathbf{p}})
\end{array}\right)
$$

(in bivector representation) and the generalized eigenfunctions of the momentum operator, are the following $[6,38,43]$ :

1) Corresponding to states of a photon with positive energy $E^{(+)}(k)=\eta k c=+p c$ (wich are consistent with the special theory of relativity [52]) the orthonormal bivectors, answering to a helicity $\lambda= \pm 1$, are

$$
\begin{aligned}
& \Phi_{\mathrm{bv} ; \mathbf{k},+1}^{(+)}(\mathbf{r}, t)=\left(\begin{array}{c}
\xi_{\mathbf{k},+1}^{(+)}(\mathbf{r}, t) \\
0
\end{array}\right)=\frac{(\mathrm{Oe}) \mathrm{e}_{+1}(\mathbf{k})}{(2 \pi)^{3 / 2}} e^{i(\mathbf{k r}-k c t)}\left(\begin{array}{l}
1 \\
0
\end{array}\right), \\
& \Phi_{\mathrm{bv} ; \mathbf{k},-1}^{(+)}(\mathbf{r}, t)=\left(\begin{array}{c}
0 \\
\eta_{\mathbf{k},-1}^{(+)}(\mathbf{r}, t)
\end{array}\right)=\frac{(\mathrm{Oe}) \mathrm{e}_{-1}(\mathbf{k})}{(2 \pi)^{3 / 2}} e^{i(\mathbf{k r}-k c t)}\left(\begin{array}{l}
0 \\
1
\end{array}\right),
\end{aligned}
$$

respectively, where (Oe) is unit of measure (Oersted) of values $\xi$ and $\eta$. 
2) Corresponding to states of a photon with negative energy $E^{(-)}(k)=-\eta k c=-p c$ (which are theoretically possible) the orthonormal bivectors, answering to the helicity $\lambda=\mu 1$, are

$$
\begin{aligned}
& \Phi_{\mathrm{bv} ; \mathbf{k},-1}^{(-)}(\mathbf{r}, t)=\left(\begin{array}{c}
\xi_{\mathbf{k},-1}^{(-)}(\mathbf{r}, t) \\
0
\end{array}\right)=\frac{(\mathrm{Oe}) \mathrm{e}_{-1}(\mathbf{k})}{(2 \pi)^{3 / 2}} e^{i(\mathbf{k r}+k c t)}\left(\begin{array}{l}
1 \\
0
\end{array}\right) \\
& \Phi_{\mathrm{bv} ; \mathbf{k},+1}^{(-)}(\mathbf{r}, t)=\left(\begin{array}{c}
0 \\
\eta_{\mathbf{k},+1}^{(-)}(\mathbf{r}, t)
\end{array}\right)=\frac{(\mathrm{Oe}) \mathrm{e}_{+1}(\mathbf{k})}{(2 \pi)^{3 / 2}} e^{i(\mathbf{k r}+k c t)}\left(\begin{array}{l}
0 \\
1
\end{array}\right)
\end{aligned}
$$

Taking into account (9), the basis vectors $\xi_{\mathbf{k}, \lambda}(\mathbf{r}, t)$ and $\eta_{\mathbf{k}, \lambda}(\mathbf{r}, t)$, also being in turn eigenfunctions of operators of the energy, momentum and helicity $\hat{\lambda}=(\hat{\mathbf{s}} \hat{\mathbf{p}}) / p$ (in vector representation), satisfy to the orthonormality relations

$$
\begin{aligned}
& \int d^{3} \mathbf{r} \xi_{\mathbf{k}^{\prime}, \lambda^{\prime}}^{+}(\mathbf{r}, t) \xi_{\mathbf{k}, \lambda}(\mathbf{r}, t)=\delta_{\lambda^{\prime} \lambda} \delta\left(\mathbf{k}^{\prime}-\mathbf{k}\right)(\mathrm{Oe})^{2} \\
& \int d^{3} \mathbf{r} \eta_{\mathbf{k}^{\prime}, \lambda^{\prime}}^{+}(\mathbf{r}, t) \eta_{\mathbf{k}, \lambda}(\mathbf{r}, t)=\delta_{\lambda^{\prime} \lambda} \delta\left(\mathbf{k}^{\prime}-\mathbf{k}\right)(\mathrm{Oe})^{2}
\end{aligned}
$$

Therefore bivectors (24) - (27) satisfy to the orthonormality relations

$$
\int d^{3} \mathbf{r}\left[\Phi_{\mathrm{bv} ; \mathbf{k}^{\prime}, \lambda^{\prime}}^{( \pm)}(\mathbf{r}, t)\right]^{+} \Phi_{\mathrm{bv} ; \mathbf{k}, \lambda}^{( \pm)}(\mathbf{r}, t)=(\mathrm{Oe})^{2} \delta_{\lambda^{\prime} \lambda} \delta\left(\mathbf{k}^{\prime}-\mathbf{k}\right)
$$

In addition, bivectors corresponding to the states with the opposite energy sign are orthogonal to each other regardless of the values $\mathbf{k}$ and $\lambda$ :

$$
\int d^{3} \mathbf{r}\left[\Phi_{\mathrm{bv} ; \mathbf{k}^{\prime}, \lambda^{\prime}}^{( \pm)}(\mathbf{r}, t)\right]^{+} \Phi_{\mathrm{bv} ; \mathbf{k}, \lambda}^{(\mu)}(\mathbf{r}, t)=0
$$

The relations (28) - (30) make it possible to decompose any vectors and bivectors in the corresponding bases:

$$
\begin{gathered}
\xi(\mathbf{r}, t)=\mathrm{E}(\mathbf{r}, t)+i \mathrm{H}(\mathbf{r}, t) \equiv \xi_{+1}^{(+)}(\mathbf{r}, t)+\xi_{-1}^{(-)}(\mathbf{r}, t)= \\
=\int B(\mathbf{k},+1) \xi_{\mathbf{k},+1}^{(+)}(\mathbf{r}, t) d^{3} \mathbf{k}+\int B(\mathbf{k},-1) \xi_{\mathbf{k},-1}^{(-)}(\mathbf{r}, t), \\
\eta(\mathbf{r}, t)=\mathrm{E}(\mathbf{r}, t)-i \mathrm{H}(\mathbf{r}, t) \equiv \eta_{-1}^{(+)}(\mathbf{r}, t)+\eta_{+1}^{(-)}(\mathbf{r}, t)= \\
=\int[B(-\mathbf{k},-1)]^{*} \eta_{\mathbf{k},-1}^{(+)}(\mathbf{r}, t) d^{3} \mathbf{k}+\int[B(-\mathbf{k},+1)]^{*} \eta_{\mathbf{k},+1}^{(-)}(\mathbf{r}, t) d^{3} \mathbf{k}, \\
\Phi_{\mathrm{bv}}^{( \pm)}(\mathbf{r}, t)=\left(\begin{array}{c}
\mathrm{E}_{\xi, \pm 1}^{( \pm)}(\mathbf{r}, t)+i \mathrm{H}_{\xi, \pm 1}^{( \pm)}(\mathbf{r}, t) \\
\mathrm{E}_{\eta, \mu 1}^{( \pm)}(\mathbf{r}, t)-i \mathrm{H}_{\eta, \mu 1}^{( \pm)}(\mathbf{r}, t)
\end{array}\right) \equiv\left(\begin{array}{c}
\xi_{ \pm 1}^{( \pm)}(\mathbf{r}, t) \\
\eta_{\mu 1}^{( \pm)}(\mathbf{r}, t)
\end{array}\right)= \\
=\int B(\mathbf{k}, \pm 1) \Phi_{\mathrm{bv} ; \mathbf{k}, \pm 1}^{( \pm)}(\mathbf{r}, t) d^{3} \mathbf{k}+\int[B(-\mathbf{k}, \mu 1)]^{*} \Phi_{\mathrm{bv} ; \mathbf{k}, \mu 1}^{( \pm)}(\mathbf{r}, t) d^{3} \mathbf{k}=
\end{gathered}
$$

From (32) - (35) it is visible that if the free electromagnetic field is initially set by means of the some classically interpreted field intensities $\mathbf{E}$ and $\mathbf{H}$, the single-photon state corresponding to this field can't be generally specified in terms of quantities $\xi$ and $\eta$, as the unobserved negative energy make a contribution in decompositions (32) - (35).

So, at the level of the postulate we can say that the singlephoton state can be described bivector (36) - (37) with a plus sign for the real photons and with the minus sign for the hypothetical photons with negative energy.

The bivector (36) - (37) also plays an important role in the description of the single-photon state.

We can denote the intensities, giving the contributions to the integrals (32) - (35) according to the following relations:

$$
\begin{aligned}
& \mathrm{E}(\mathbf{r}, t)=\mathrm{E}_{\xi,+1}^{(+)}(\mathbf{r}, t)+\mathrm{E}_{\xi,-1}^{(-)}(\mathbf{r}, t)=\mathrm{E}_{\eta,-1}^{(+)}(\mathbf{r}, t)+\mathrm{E}_{\eta,+1}^{(-)}(\mathbf{r}, t), \\
& \mathrm{H}(\mathbf{r}, t)=\mathrm{H}_{\xi,+1}^{(+)}(\mathbf{r}, t)+\mathrm{H}_{\xi,-1}^{(-)}(\mathbf{r}, t)=\mathrm{H}_{\eta,-1}^{(+)}(\mathbf{r}, t)+\mathrm{H}_{\eta,+1}^{(-)}(\mathbf{r}, t)
\end{aligned}
$$

Then from (36), (38) important connections follow:

$$
\begin{gathered}
\mathrm{E}_{\xi, \mu 1}^{(\mu)}(\mathbf{r}, t)=\mathrm{E}_{\eta, \mu 1}^{( \pm)}(\mathbf{r}, t), \quad \mathrm{H}_{\xi, \mu 1}^{(\mu)}(\mathbf{r}, t)=\mathrm{H}_{\eta, \mu 1}^{( \pm)}(\mathbf{r}, t), \\
{\left[\xi_{-1}^{(-)}(\mathbf{r}, t)\right]^{*}=\eta_{-1}^{(+)}(\mathbf{r}, t), \quad\left[\eta_{+1}^{(-)}(\mathbf{r}, t)\right]^{*}=\xi_{+1}^{(+)}(\mathbf{r}, t)}
\end{gathered}
$$

Together with (43) - (44) associating with the decompositions (33), (35), the relations (40), (41) give [6, 38, 43] the superposition principle for the intensities $\mathbf{E}$ and $\mathbf{H}$ :

$$
\begin{aligned}
& \mathrm{E}(\mathbf{r}, t)=\mathrm{E}_{\xi,+1}^{(+)}(\mathbf{r}, t)+\mathrm{E}_{\eta,-1}^{(+)}(\mathbf{r}, t), \\
& \mathrm{H}(\mathbf{r}, t)=\mathrm{H}_{\xi,+1}^{(+)}(\mathbf{r}, t)+\mathrm{H}_{\eta,-1}^{(+)}(\mathbf{r}, t)
\end{aligned}
$$

Using specified bivectors, it is possible to write the spatial density distribution of photon energy in the state (37):

$$
\begin{gathered}
\rho_{E}^{( \pm)}(\mathbf{r}, t) \equiv \frac{1}{8 \pi}\left[\Phi_{\mathrm{bv}}^{( \pm)}(\mathbf{r}, t)\right]^{+} \Phi_{\mathrm{bv}}^{( \pm)}(\mathbf{r}, t)= \\
=\frac{1}{8 \pi}\left\{\left[\xi_{ \pm 1}^{( \pm)}(\mathbf{r}, t)\right]^{+} \xi_{ \pm 1}^{( \pm)}(\mathbf{r}, t)+\left[\eta_{\mu 1}^{( \pm)}(\mathbf{r}, t)\right]^{+} \eta_{\mu 1}^{( \pm)}(\mathbf{r}, t)\right\} \equiv \\
=\frac{1}{8 \pi}\left\{\left[\mathbf{E}_{\xi, \pm 1}^{( \pm)}(\mathbf{r}, t)\right]^{2}+\left[\mathbf{H}_{\xi, \pm 1}^{( \pm)}(\mathbf{r}, t)\right]^{2}+\left[\mathbf{E}_{\eta, \mu 1}^{( \pm)}(\mathbf{r}, t)\right]^{2}+\left[\mathbf{H}_{\eta, \mu 1}^{( \pm)}(\mathbf{r}, t)\right]^{2}\right\}
\end{gathered}
$$

This energy density is to be distinguished from the "classical" energy density, which, obviously, has the form

$$
\begin{aligned}
& \rho_{E}^{(c l)}(\mathbf{r}, t) \equiv \frac{1}{8 \pi}\left\{[\mathbf{E}(\mathbf{r}, t)]^{2}+[\mathbf{H}(\mathbf{r}, t)]^{2}\right\}= \\
& \equiv \frac{1}{16 \pi}\left[\Phi_{\mathrm{bv}}^{(+)}(\mathbf{r}, t)+\Phi_{\mathrm{bv}}^{(-)}(\mathbf{r}, t)\right]^{+}\left[\Phi_{\mathrm{bv}}^{(+)}(\mathbf{r}, t)+\Phi_{\mathrm{bv}}^{(-)}(\mathbf{r}, t)\right]
\end{aligned}
$$

From the practical point of view, the construction of the bivector (36) - (37) isn't so much important in itself, how the finding of the coefficients $B(\mathbf{k}, \lambda)$ is important in the case, when the single-photon state is defined to be corresponding to the state of the electromagnetic field, initially given by means of the classical intensities $\mathbf{E}$ and $\mathbf{H}$. Further, composing, for 
example, the vector $\xi=\mathbf{E}+i \mathbf{H}$ and using the orthonormality relations (28), (30), it is possible to calculate the coefficients

$$
\begin{gathered}
B(\mathbf{k}, \pm 1)=\frac{1}{(\mathrm{Oe})^{2}} \int d^{3} \mathbf{r}\left[\xi_{\mathbf{k}, \pm 1}^{( \pm)}(\mathbf{r}, t)\right]^{+} \xi(\mathbf{r}, t)= \\
=\frac{1}{(\mathrm{Oe})^{2}} \int d^{3} \mathbf{r}\left[\Phi_{\mathrm{bv} ; \mathbf{k}, \pm 1}^{( \pm)}(\mathbf{r}, t)\right]^{+} \Phi^{( \pm)}(\mathbf{r}, t)
\end{gathered}
$$

Then with these coefficients we can calculate the coefficients $b(\mathbf{k}, \lambda)$ appearing in (1) by the formula

$$
b(\mathbf{k}, \lambda)=\frac{(\mathrm{Oe})}{\sqrt{8 \pi \eta k c}} B(\mathbf{k}, \lambda)
$$

The introduction of these coefficients most fully implements the principle of correspondence. Indeed, according to (7), (24) - (27), (34), (37), (47), based on the classical electrodynamics, the total photon energy can be written as

$$
\begin{aligned}
E & =E^{(+)}=\int d^{3} \mathbf{r} \rho_{E}^{(c l)}(\mathbf{r}, t)=\frac{(\mathrm{Oe})^{2}}{8 \pi} \int d^{3} \mathbf{k} \sum_{\lambda}|B(\mathbf{k}, \lambda)|^{2}= \\
& =\int d^{3} \mathbf{k} \sum_{\lambda}|b(\mathbf{k}, \lambda)|^{2} \eta k c=\int d^{3} \mathbf{k} \rho_{P}^{( \pm)}(\mathbf{k}) \eta k c
\end{aligned}
$$

The integration of density $\rho_{E}^{( \pm)}(\mathbf{r}, t)$ yields just the same result.

On the other hand, the same result is obtained if we use the purely quantum-mechanical formula of calculating of the energy mean value in the state (1) and the Hamilton operator (17) (and also the equation (16), and relations (30)):

$$
\begin{aligned}
& \overline{E^{( \pm)}} \equiv\left\langle\Psi^{( \pm)}\left|\hat{H}_{\mathrm{bv}}\right| \Psi^{( \pm)}\right\rangle=\left\langle\Psi^{( \pm)} \mid i \eta \frac{\partial \Psi^{( \pm)}}{\partial t}\right\rangle= \\
= & \int( \pm \eta k c) \sum_{\lambda}|b(\mathbf{k}, \lambda)|^{2} d^{3} \mathbf{k}=\int E^{( \pm)}(k) \rho_{P}^{( \pm)}(\mathbf{k}) d^{3} \mathbf{k},
\end{aligned}
$$

what coincides with (52) for positive energy.

To conclude this paragraph we mention that from (16) the continuity equation follows [6, 38, 43] for density of probability $\rho_{P}^{( \pm)}(\mathbf{r}, t)$ and of stream density $\mathbf{j}_{P}^{( \pm)}(\mathbf{r}, t)$ of probability to find the photon in the vicinity of the point $\mathbf{r}$ in a time point $t$ :

where

$$
\frac{\partial \rho_{P}^{( \pm)}(\mathbf{r}, t)}{\partial t}+\operatorname{div} \mathbf{j}_{P}^{( \pm)}(\mathbf{r}, t)=0
$$

$$
\begin{gathered}
\rho_{P}^{( \pm)}(\mathbf{r}, t)=\left[\Psi^{( \pm)}(\mathbf{r}, t)\right]^{+} \Psi^{( \pm)}(\mathbf{r}, t), \\
\mathbf{j}_{P}^{( \pm)}(\mathbf{r}, t)=c\left[\Psi^{( \pm)}(\mathbf{r}, t)\right]^{+} \hat{\alpha}_{\mathrm{bv}} \Psi^{( \pm)}(\mathbf{r}, t) .
\end{gathered}
$$

Although for arbitrary coefficients $b(\mathbf{k}, \lambda)$ the (55) doesn't possess of relativistic invariance property, nevertheless it can be shown that this equation is relativistic invariant $[38,43]$ for the case of plane monochromatic waves.

\section{Modeling OF SHORT-Puls LaSer RADiation}

On the basis of the above stated general method of construction of wave function of a free photon in coordinate representation in $[1-6,46,47]$ the most important wave packet in scientific and methodical aspects with Gaussian momentum distribution is considered, namely with the coefficients

$$
\begin{gathered}
b(\mathbf{k}, \pm 1)=[b(-\mathbf{k}, \mu 1)]^{*}= \\
=\sqrt{\frac{\alpha^{3}}{2 \pi \sqrt{\pi}}} \exp \left[-\frac{\alpha^{2}}{2}\left(k_{x}^{2}+k_{y}^{2}+\left(k_{z} \mu k_{0}\right)^{2}\right)-i \mathbf{k r}_{0}\right],
\end{gathered}
$$

where parameters $\mathbf{k}_{0}=\left(0,0, k_{0}\right), \quad \mathbf{r}_{0}=\left(x_{0}, y_{0}, z_{0}\right), \quad \alpha$ characterize the average values and the dispersions of the corresponding physical quantities in the state of a photon (1) and satisfy the normalization condition (2).

Parameterization (58) answers to the state of a photon with zero average helicity as the $\lambda= \pm 1$ are presented in (58) with equal probability. All characteristics can be broken into two categories: momentum-energy and space-time. According to quantum mechanics, values of these categories characteristics answer to the corresponding uncertainty relations. New here, compared to the particles with mass, is the fact that the values of characteristics of the second category essentially can depend on "choice" of vectors $\mathbf{e}_{\lambda}(\mathbf{k})$.

Requirements (8) - (13) are satisfied, e.g., for the following polarization vectors $[1,3-6,46,47]$ :

$$
\begin{aligned}
& e_{I}(\mathbf{k})=\left(\begin{array}{c}
1-(1-\cos \theta) \cos ^{2} \varphi \\
-(1-\cos \theta) \sin \varphi \cos \varphi \\
-\sin \theta \cos \varphi
\end{array}\right) \\
& e_{I I}(\mathbf{k})=\left(\begin{array}{c}
-(1-\cos \theta) \sin \varphi \cos \varphi \\
\cos \theta+(1-\cos \theta) \cos ^{2} \varphi \\
-\sin \theta \sin \varphi
\end{array}\right) \text { at } 0 \leq \theta \leq \frac{\pi}{2} \\
& e_{I}(\mathbf{k})=\left(\begin{array}{c}
1-(1+\cos \theta) \cos ^{2} \varphi \\
-(1+\cos \theta) \sin \varphi \cos \varphi \\
\sin \theta \cos \varphi
\end{array}\right) \\
& e_{I I}(\mathbf{k})=\left(\begin{array}{c}
(1+\cos \theta) \sin \varphi \cos \varphi \\
\cos \theta-(1+\cos \theta) \cos ^{2} \varphi \\
-\sin \theta \sin \varphi
\end{array}\right) \text { at } \frac{\pi}{2}<\theta \leq \pi
\end{aligned}
$$

where the Cartesian components of the corresponding vectors in the configuration space are specified, expressed in terms of the spherical coordinates of vector $\mathbf{k}$ in momentum space.

Applying (1), (58) and quantum-mechanical formula of calculation of average value of physical quantity $F$, the operator of which is equal $\hat{F}$, namely

$$
\overline{F^{( \pm)}} \equiv\left\langle\Psi^{( \pm)} \mid \hat{F} \Psi^{( \pm)}\right\rangle=\left\langle\sum_{\lambda^{\prime}} \Psi_{\lambda^{\prime}}^{( \pm)} \mid \hat{F} \sum_{\lambda} \Psi_{\lambda}^{( \pm)}\right\rangle
$$

where $\Psi_{\lambda}^{( \pm)}$at the $\lambda= \pm 1$ are defined by corresponding terms 
of the formula (1), we find in state (1) the average values of momentum projections and the energy of photon, respectively with positive and negative spectrum of its energy:

$$
\begin{gathered}
\overline{p_{x}^{( \pm)}}=\overline{p_{y}^{( \pm)}}=0, \overline{p_{z}^{( \pm)}}= \pm \eta k_{0}, \\
\overline{E^{( \pm)}}= \pm \eta k_{0} c\left[\left(1+\frac{1}{2 \alpha^{2} k_{0}^{2}}\right) \operatorname{erf}\left(\alpha k_{0}\right)+\frac{\exp \left(-\alpha^{2} k_{0}^{2}\right)}{\alpha k_{0} \sqrt{\pi}}\right]
\end{gathered}
$$

Then uncertainties of the photon momentum projections in state (1) are reduced to formulas

$$
\Delta p_{x} \equiv \sqrt{D_{p_{x}}}=\frac{\eta}{\alpha \sqrt{2}}, \quad \Delta p_{y}=\Delta p_{z}=\frac{\eta}{\alpha \sqrt{2}},
$$

and according to (6) the simplest, namely Gaussian, form of the momentum distribution in the state (1) is obtained

$$
\begin{gathered}
\rho_{P}^{( \pm)}(\mathbf{k})=|b(\mathbf{k}, \pm 1)|^{2}+|b(-\mathbf{k}, \mu 1)|^{2}= \\
=\frac{\alpha^{3}}{\pi \sqrt{\pi}} \exp \left[-\alpha^{2}\left(k_{x}^{2}+k_{y}^{2}+\left(k_{z} \mu k_{0}\right)^{2}\right)\right] .
\end{gathered}
$$

The average values of the coordinates of the detection point of the photon in state (1) get the following

$$
\overline{x^{( \pm)}}=x_{0} ; \quad \overline{y^{( \pm)}}=y_{0} ; \quad \overline{z^{( \pm)}}=z_{0} \pm c t \overline{n_{z}^{( \pm)}}
$$

where

$$
\overline{n_{z}^{( \pm)}}=\left[\left(1-\frac{1}{2 \alpha^{2} k_{0}^{2}}\right) \operatorname{erf}\left(\alpha k_{0}\right)-\frac{\exp \left(-\alpha^{2} k_{0}^{2}\right)}{\alpha k_{0} \sqrt{\pi}}\right] .
$$

In $[1-6,46,47]$ the analysis is carried out of the evolution of the considered wave packet by calculation of an electric intensity taking into account (1), (42), (51), (59) - (62). Practically, not equal to zero in this case is only the projection of electric field intensity $E_{x}$ which characterizes, definitely, a space probability density. For the wave packet corresponding to the duration $\tau_{\text {rad }}=80 \mathrm{fs}$ of the laser radiation with the central wavelength of 10 microns and with the parameter $\alpha=0.00169$ calculated by means of the uncertainty relation for energy and time $\Delta E \tau_{\text {rad }} \cong \eta$ (see, for example [53]), the numerical analysis shows that this wave packet is quickly extended out in all directions symmetrically to the $z$ axis, and so that its periphery significantly lags behind the central part moving almost with speed $c$ according to (69). Thus, there is a transformation of an initial "spherical" form of the wave packet into the some "conical" shape, reminding the VavilovCherenkov radiation.

\section{The Main Formula of Wave-Particle Duality AND NATURE OF PHOTON}

In our view, the created photon quantum mechanics essentially removes the wave-particle duality problem. Its main formula is expressed as follows [1, 4-6, 43, 46, 47, 53]:

1. At interaction the photons and particles behave as a corpuscles, transferring and transmitting to other particles the certain quantity as dynamic characteristics (momentum, energy, angular momentum), and "internal" (mass, electric charge, spin, etc.). Particularly, the transfer takes place at hit of a photon or particle in quite dot detector (or a point on the screen) with coordinate $\mathbf{r}$ at time point $t$. The fact of hit of "all particle entirely" in the dot detector is characteristic for a corpuscle, but not for some real wave.

2 . However photons and microparticles propagate in space by "wave rules", that is their distribution in space is described by the wave function. In particular, density of probability of detection in space of the nonrelativistic particle with a nonzero mass is postulated by a formula $\rho(\mathbf{r}, t)=|\Psi(\mathbf{r}, t)|^{2}$, and a photon by (3). This probability density also causes the hit of a photon and particle in the dot detector. A characteristic interferential picture on the screen corresponds to distribution of $\rho(\mathbf{r}, t)$ along the screen.

Nevertheless, electromagnetic radiation even in the case of waves small lengths and obviously expressed "corpuscular properties", is impossible to consider as a stream of the certain "created", "dot" particles, similar to the massive particles. In our view, the photon is a quasi-particle, and light is a result of the propagation of a spin wave in physical vacuum, the structure and nature of which have to be considered at the Planck distance $[6,43,54]$. This question is closely related to the structure of the leptons and other fundamental particles on the same distances. According to [55-57] the center of an electron is extreme maximon, that is the quantum nonsingular object creating round itself an extreme Kerr-Newman metric. It has spin $s=1 / 2$ and approximately Planck mass, charge and radius. For most observed phenomena involving photons it is possible to give the following interpretation of their propagation in vacuum. In the photon propagation the middleordered (in time and space) alternate spins flip occurs (during the Planck time $T_{P}$ for each flip, see Fig.1a) of virtual vacuum extreme maximons (or, the same, friedmons, planckeons), which creates the effect of the spin wave, and in "macroscopic scale" produce manifestation the corpuscular-wave properties of photons. However due to the vector dominance also exhibited by the photons, their propagation in vacuum can be associated also with other, more complex virtual processes.

\section{THE YOUNG'S INTERFERENCE EXPERIMENT}

It is known that when problem of wave-particle duality is discussed, then for example in experiment such as the Young's experiment, to explain the wave properties of particles having mass, the wave function in the coordinate representation is attracted. The similar picture of diffraction and interference observed with the light is explained, appealing to the classical electrodynamics based on Maxwell's equations. So, in the case of Young's experiment the explanation of the interference result is reduced to establishment of the phases difference of two monochromatic waves emitted by the slits 1 and 2 (see Fig. 1b), entering the observation point $P$ on second screen. 


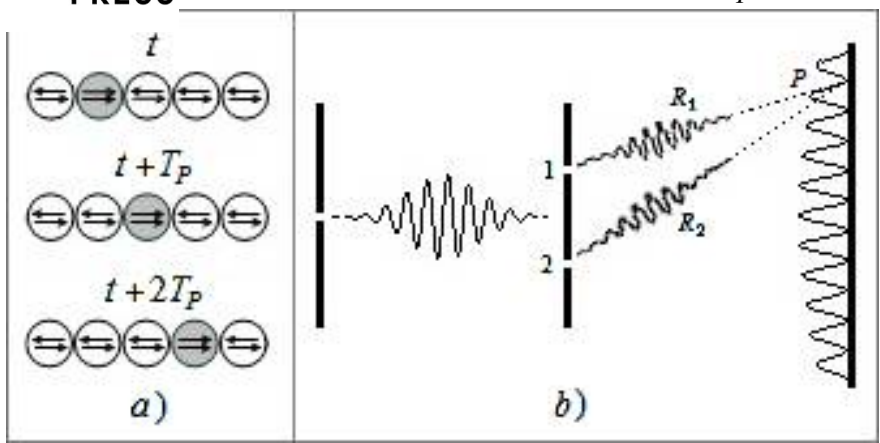

Fig. 1. Propagation of the photon in space: a) spins flip wave in physical vacuum at Planck distances; b) penetration of the wave function of a photon at a time through two slits in Young's experiment.

In [1] the idea was introduced that similar phase $\varphi=\mathbf{k r}-k c t$ is available in each term of the photon wave function (1). If the radiation is more or less monochromatic, then in the expression for the probability density (3), obviously, arises the member proportional to the cosine of the phases difference $\delta=\varphi_{1}-\varphi_{2}=k\left(r_{1}-r_{2}\right)$ of these two waves (emitted by the slits 1 and 2 ). This also provides an explanation for the occurrence of interference fringes, similarly to the explanation of classical electrodynamics.

Let us consider this idea in more detail. We now set, instead of (58), the coefficients $b(\mathbf{k}, \pm 1)$ equal to

$$
b(\mathbf{k}, \pm 1)=b(k, \pm 1)=\frac{\alpha}{\sqrt{\pi}} \exp \left[-\alpha^{2}\left(k-k_{0}\right)^{2}\right],
$$

which corresponds to the propagation of a spherical wave describing the photon state with an average zero momentum vector and the average modulus equal $\eta k_{0}$. Although (71) does not satisfy the normalization (2) at account (7), the choice of (71) realizes the delta-shaped function for allowing to clearly select a monochromatic wave in the decomposition (1) on plane waves and, in addition, to obtain a rather simple analytical expression for the photon wave function.

Let's decompose the $e^{i \mathbf{k r}}$ in (1), on spherical functions:

$$
e^{i \mathbf{k r}}=\sum_{\lambda=0}^{\infty} \sum_{m=-\lambda}^{\lambda} i^{\lambda} j_{\lambda}(k r) Y_{\lambda m}^{*}(\theta, \varphi) Y_{\lambda m}\left(\theta_{r}, \varphi_{r}\right)
$$

where $\theta_{r}, \varphi_{r}$ determines the radius vector in spherical coordinate system of the configuration space, $j_{\lambda}(k r)$ is spherical Bessel functions; in particular,

$$
j_{0}(k r)=\sin (k r) /(k r) .
$$

Substituting the polarization vectors (59) - (62), and also (72), in (1) and using the orthonormality of the spherical functions $Y_{\lambda m}(\theta, \varphi)$, we obtain

$$
\Psi( \pm)(\mathbf{r}, t)=
$$

$=\left(\begin{array}{l}1 \\ 1\end{array}\right) \frac{1}{r \sqrt{4 \pi}} \int_{0}^{\infty} k b(k, \pm 1) e^{\mu i k c t}\left(\begin{array}{c}\sin (k r)-i \cos (k r) \cos \left(\theta_{r}\right) \\ 0 \\ i \cos (k r) \sin \left(\theta_{r}\right) e^{i \varphi_{r}}\end{array}\right) d k$, where we neglected the terms proportional $1 / r^{2}$, keeping in mind the radiation in the wave zone. In (74) the coefficient $b(\mathbf{k}, \pm 1)$ has not yet been specified in a concrete form. Substituting (71) instead of it, at $\alpha \rightarrow \infty$, that finally corresponds to the selection of the monochromatic wave by this method, we find

$$
\Psi( \pm)(\mathbf{r}, t)=\left(\begin{array}{l}
1 \\
1
\end{array}\right) \frac{k_{0} e^{\mu i k_{0} c t}}{r \sqrt{4 \pi}}\left(\begin{array}{c}
\sin \left(k_{0} r\right)-i \cos \left(k_{0} r\right) \cos \left(\theta_{r}\right) \\
0 \\
i \cos \left(k_{0} r\right) \sin \left(\theta_{r}\right) e^{i \varphi_{r}}
\end{array}\right) .
$$

Multiplying (75) by the necessary factor, independent of the coordinates and time, we can restore the correct dimension of the photon wave function, which is disturbed by the parameterization (71). Taking into account it, let us write down the wave function of a photon passing "through both holes" at the first screen in Young's experiment, as the sum of two terms, each of which has the form (75):

$$
\begin{gathered}
\Psi( \pm)(\mathbf{r}, t)=\Psi_{1}^{( \pm)}(\mathbf{r}, t)+\Psi_{2}^{( \pm)}(\mathbf{r}, t)= \\
=\Psi_{1}^{( \pm)}\left(\mathbf{r}_{1}+\frac{\mathbf{d}}{2}, t\right)+\Psi_{2}^{( \pm)}\left(\mathbf{r}_{2}-\frac{\mathbf{d}}{2}, t\right)= \\
=\left(\begin{array}{l}
1 \\
1
\end{array}\right) \frac{A e^{\mu i k_{0} c t}}{r_{1}}\left(\begin{array}{c}
\sin \left(k_{0} r_{1}\right)-i \cos \left(k_{0} r_{1}\right) \cos \left(\theta_{r 1}\right) \\
0 \\
i \cos \left(k_{0} r_{1}\right) \sin \left(\theta_{r 1}\right)
\end{array}\right)+ \\
+\left(\begin{array}{c}
1 \\
1
\end{array}\right) \frac{A e^{\mu i k_{0} c t}}{r_{2}}\left(\begin{array}{c}
\sin \left(k_{0} r_{2}\right)-i \cos \left(k_{0} r_{2}\right) \cos \left(\theta_{r 2}\right) \\
0 \\
i \cos \left(k_{0} r_{2}\right) \sin \left(\theta_{r 2}\right)
\end{array}\right),
\end{gathered}
$$

where $A$ is the real normalization constant; the angles $\theta_{r 1}$, $\theta_{r 2}$ are counted from the spatial axis, now directed along the line connecting the both holes (in the first screen) connected by the vector $\mathbf{d}$; the angles $\varphi_{r 1}, \varphi_{r 2}$ are set equal to zero, and $r_{1}$ и $r_{2}$ are the distances from the holes to the interference observation point $\mathrm{P}$ on the second screen (apart from the first screen at a distance $\lambda$ ).

Having written down the probability density of detection of a photon according to (3), it is easy to verify that the interference result is determined by the interference term, which after transformations is reduced to the form

$$
\begin{gathered}
\rho_{\text {int }}=\frac{2 A^{2}}{r_{1} r_{2}}\left[\sin \left(k_{0} r_{1}\right) \sin \left(k_{0} r_{2}\right)+\right. \\
\left.+\cos \left(k_{0} r_{1}\right) \cos \left(k_{0} r_{2}\right) \cos \left(\theta_{r 1}-\theta_{r 2}\right)\right] .
\end{gathered}
$$

In the geometry of the Young's double-slit experiment, which is usually considered in classical electrodynamics, it is assumed that $r_{1}+r_{2}=2 \lambda, r_{2}-r_{1}=\Delta$ where $\Delta$ is the optical path difference (in vacuum or air) of the rays emanating from both holes. In the same approximation, then, approximate equations are used, namely: $\cos \left(\theta_{r 1}-\theta_{r 2}\right) \approx 1, r_{1} r_{2} \approx \lambda^{2}$, $\Delta \approx z d / \lambda$, where $z$ is the coordinate of the point $\mathrm{P}$ on the 
second screen, measured from the (average) symmetry line of the interference pattern. Taking these relations into account, the interference term (78) assumes exactly the same form as in classical electrodynamics:

$$
\rho_{\text {int }}=\frac{2 A^{2}}{\lambda^{2}} \cos \left(k_{0} \Delta\right)=\frac{2 A^{2}}{\lambda^{2}} \cos \delta,
$$

where $\delta=2 \pi \Delta / \lambda_{0}$ is the phase difference of two interfering rays from the point of view of classical electrodynamics, $k_{0}=2 \pi / \lambda_{0}$ is the wave number of the corresponding (almost) monochromatic radiation with the wavelength $\lambda_{0}$.

Thus, introducing in consideration the photon wave function in the coordinate representation, we have the opportunity to explain the wave phenomena on a uniform basis for all quantum particles. This especially becomes relevant when an experiment involving photons emitted obviously alone (for the first time single photons have been reliably fixed in [58]).

\section{CONCLUSION}

The results of our modeling of photon wave packet propagation allow to illustrate the possibility of a singlephoton approach to the description of electromagnetic phenomena. In particular, it appears that those aspects of interference and diffraction such as the interference pattern of Young's double-slit experiment, which were described in the language of classical electrodynamics, obviously can be described in the language of quantum mechanics without the involvement of the apparatus of second quantization of the electromagnetic field. This significantly expands the scope of "ordinary" quantum mechanics and considerably reduces the problem of wave-particle duality in the present level of our knowledge. However, in our view, the photon is a quasiparticle actually, and light is a result of the propagation of a spin-flip wave in physical vacuum, the structure and nature of which have to be considered at the Planck distance. Perhaps, this circumstance will allow to look from the new point of view at the such problems as the quantum entanglement of particles and their nonlocality of the interactions.

\section{REFERENCES}

[1] A.P. Davydov, T.P. Zlydneva, "The Young's interference experiment in the light of the single-photon modeling of the laser radiation", in Information Technologies in Science, Management, Social Sphere and Medicine (ITSMSSM 2016), pp. 208-215, 2016. URL: http://www.atlantis-press.com/php/pub.php?publication=itsmssm-16 (accessed 21 October 2017) DOI: 10.2991/itsmssm-16.2016.100.

[2] A.P. Davydov, "Jevoljucija v prostranstve i vo vremeni volnovogo paketa fotona femtosekundnogo izluchenija s tochki zrenija kvantovoj mehaniki", in Tezisy dokl. XLIII vnutrivuz. nauch. konf. prepodavatelej MaGU "Sovremennye poblemy nauki i obrazovanija", pod red. P.Ju. Romanova i E.M. Razinkinoj. Magnitogorsk: Izd-vo Magnitogorsk. gos. un-ta, pp. 269-270, 2005.

[3] A.P. Davydov, "Modelirovanie rasprostranenija v trehmernom prostranstve volnovogo paketa fotona", in Aktual'nye poblemy sovremennoj nauki, tehniki i obrazovanija: materially 73-j mezhdun. nauch.-tehnich. konf. Magnitogorsk: Izd-vo Magnitogorsk. gos. tehn. un-ta im. G.I. Nosova, vol. 3, pp. 133-137, 2015.

[4] A.P. Davydov, T.P. Zlydneva, "Odnofotonnyj podhod k modelirovaniju korotkoimpul'snogo lazernogo izluchenija”, in Vestnik nauki i obrazovanija Severa-Zapada Rossii: jelektronnyj zhurnal, vol. 1, № 4, 2015. URL: http://vestnik-nauki.ru/ (accessed 21 October 2017).

[5] A. Davydov, T. Zlydneva, "Modeling of short-pulse laser radiation in terms of photon wave function in coordinate representation" [Electronic resource], in Instrumentation engineering, electronics and telecommunications - 2015: Paper book of the International Forum IEET-2015, pp. 51-63, Izhevsk: Publishing House of Kalashnikov ISTU, 208 p, 7 MB, 2016. URL: http://pribor21.istu.ru/proceedings/IEET2015.pdf (accessed 21 October 2017).

[6] A.P. Davydov, "Volnovaja funkcija fotona $v$ koordinatnom predstavlenii": monografija. Magnitogorsk: Izd-vo Magnitogorsk. gos. techn. un-ta im. G.I. Nosova, 2015. 180 p.

[7] L. Landau and R. Peierls, "Quantenelectrodynamik im Konfigurationsraum”, Zeit. F. Phys, vol. 62, pp. 188-198, 1930.

[8] H.A. Kramers, Quantum Mechanics, Amsterdam: North- Holland, 1958 (original edn 1937).

[9] T.D. Newton and E.P. Wigner. "Localized states for elementary particles", Reviews of Modern Physics, vol. 21, pp. 400-406, 1949.

[10] D. Bohm, Quantum Theory, Constable, London, 1954.

[11] E. A. Power, Introductory Quantum Electrodynamics, Longmans, London, 1964.

[12] V.B. Berestetskii, E.M. Lifshitz, and L. P. Pitaevskii, Quantum electrodynamics, 2nd ed. (Pergamon Press Ltd., NY, 1982).

[13] A.I. Ahiezer and V. B. Beresteckij, Kvantovaja jelectrodynamika, M.: Nauka, 1981.

[14] V.G. Levich, Ju. A.Vdovin, and V. A. Mjamlin, Kurs teoreticheskoj fiziki, Tom II, M.: Nauka, 1971.

[15] I. Bialynicki-Birula, "On the Wave Function of the Photon", Acta Phys. Pol. A, vol. 86, pp. 97-116, 1994.

[16] M. Mandel, E. Wolf, Optical coherence and quantum optics, Cambridge University Press, 1995.

[17] J. E. Sipe, "Photon wave functions", Physical Review A, vol. 52, pp. 1875-1883, 1995.

[18] A.P. Davydov, "Kvantovaja mehanika fotona", in Tezisy dokl. XXXIII nauch. konf. prepodavatelej MGPI "NAUKA I SHKOLA". Magnitogorsk: Izd-vo Magnitogorsk. gos. ped. in-ta, pp. 206-207, 1995.

[19] R.Y. Chiao, P.G. Kwiat, and A.M. Steinberg, "Quantum non-locality in two-photon experiments at Berkeley", Quantum and Semiclassical Optics: Journal of the European Optical Society, Part B, vol. 7, № 3, pp. 259-278, 1995.

[20] M.O. Scully and M.S. Zubairy, Quantum Optics. Cambridge Univ. Press, 1997, Chs. 1 and 21.

[21] V.I. Fushhich and A.G. Nikitin, "O novyh i staryh simmetrijah uravnenij Maksvella i Diraka”, W.I. Fushchych. Scientific Works, vol. 2. Kyiv: Editor Vyacheslav Boyko, 2000, pp. 233-278.

[22] A.K. Zvezdin, "Kvantovaja mehanika plenennyh fotonov, opticheskie microrezonatory, volnovody, fotonnye kristally”, Priroda, № 10, pp. 1223, 2004.

[23] E.E. Zanimonskij and Ju.P. Stepanovskij, "Precessija spina fotonov i geometricheskie fazy", Visnik HNU, № 914, Serija Fizika, Vyp. 13, pp. 36-39, 2010.

[24] A.T. Gavrilin, "Ob amplitude verojatnosti mestopolozhenija fotona", Vestnik Nizhegorodskogo universiteta im. N.I. Lobachevskogo, № 6 (1), 2011, pp. 70-74.

[25] D.N. Klyshko, "Kvantovaja optika: kvantovye, klassicheskie i metafizicheskie aspekty”, UFN, vol. 164, № 11, pp. 1187-1214, 1994.

[26] N. Gisin, G. Ribordy, W. Tittel, H. Zbinden, "Quantum cryptography”, Rev. Mod. Phys, vol. 74, № 1, pp. 145-190, 2002.

[27] V. Scarani, H. Bechmann-Pasquinucci, N.J. Cerf et al, "The security of practical quantum key distribution”, Rev. Mod. Phys., vol. 81, pp. 13011350, 2009.

[28] J. Fulconis, O. Alibart, J.L. O’Brien, W.J. Wadsworth, J.G. Rarity, "Photonic crystal fibre source of photon pairs for quantum information processing", arXiv preprint quant-ph/0611232, 2006.

[29] Liang Cui, Xiao-Ying Li, Hai-Yang Fan, Lei Yang, Xiao-Xin Ma, "Photonic Crystal Fiber Source of Quantum Correlated Photon Pairs in 
the 1550 nm, Telecom Band”, Chin. Phys. Lett, vol. 26, Iss. 4, 044209, 2009.

[30] E. Waks, K. Inoue, W.D. Oliver, E. Diamanti, Y. Yamamoto, "High efficiency photon number detection for quantum information processing", ArXiv: quant-ph/0308054, 2003.

[31] I. Procházka, K. Hamal, B. Sopko, "Recent achievements in single photon detectors and their applications”, J. Mod. Opt., vol. 51, № 9-10, pp. 1289-1313, 2004.

[32] E.J. Gansen, M.A. Rowe, M.B. Greene, D. Rosenberg, T.E. Harvey, M.Y. Su, R.H. Hadfield, S.W. Nam, R.P. Mirin, "Photon-numberdiscriminating detection using a quantum-dot, optically gated, fieldeffect transistor", Nature Photonics, vol. 1, № 10, pp. 585-588, 2007.

[33] L. A. Jiang, E. A. Dauler, J. T. Chang, "Photon-number-resolving detector with 10 bits of resolution", Phys. Rev. A.. vol. 75, Iss. 6, $062325,2007$.

[34] B.E. Kardunal, Z.L. Yuan, A.J. Shields, "An avalanche-photodiodebased photon-number-resolving detector", Nature Photonics, vol. 2, № 7, pp. 425-428, 2008.

[35] I. Bialynicki-Birula. "The Photon Wave Function", in Coherence and Quantum Optics VII, edited by J. H. Eberly, L. Mandel, and E. Wolf (Plenum Press, New York, 1996), pp. 313-323.

[36] I. Bialynicki-Birula, "Photon Wave Function", in Progress in Optics, edited by E. Wolf (North-Holland, Elsevier, Amsterdam, 1996), vol. XXXVI, pp. 248-294.

[37] M. Hawton, "Photon wave functions in a localized coordinate space basis", Phys. Rev. A, vol. 59, pp. 3223-3227, 1999.

[38] A.P. Davydov, Volnovaja funkcija fotona $v$ koordinatnom predstavlenii", in Vestnik MaGU: Periodicheskij nauchnyj zhurnal, Vyp. 5, Estestvennye nauki. Magnitogorsk: Izd-vo Magnitogorsk. gos. un-ta, pp. 235-243, 2004.

[39] B.J. Smith and M.G. Raymer, "Photon wave functions, wave-packet quantization of light, and coherence theory," New J. Phys., vol. 9, pp. 414-448, 2007.

[40] J. Cugnon, "The Photon Wave Function", Open Journal of Microphysics, vol. 1, № 3, pp. 41-52, 2011.

[41] E. Brainis, "Quantum imaging with $N$-photon states in position space", Optics Express, vol. 19(24), pp. 24228-24240, 2011.

[42] P. Saari, "Photon localization revisited," in Quantum Optics and Laser Experiments, Edited by S.Lyagushyn, S., InTech, Open Access Publisher, Croatia, pp. 49-66, 2012.

[43] A.P. Davydov, "Kvantovaja mehanika fotona: volnovaja funkcija v koordinatnom predstavlenii", Jelektromagnitnye volny i jelektronnye sistemy, vol. 20, № 5, pp. 43-61, 2015.

[44] A. P. Davydov, T. P. Zlydneva. "O reljativistskoj invariantnosti uravnenija nepreryvnosti v kvantovoj mehanike fotona", Mezhdunarodnyj nauchno-issledovatel'skij zhurnal, № 4 (46), Part 6, pp. 134-137, 2016 DOI: 10.18454/IRJ.2016.46.145.

[45] A. P. Davydov, T. P. Zlydneva. "O volnovoj funkcii fotona v koordinatnom i impul'snom predstavleniyah", Mezhdunarodnyj nauchno-issledovatel'skij zhurnal, № 11 (53), part 4, pp. 152-155 DOI: 10.18454/IRJ.2016.53.104.

[46] A. P. Davydov, T. P. Zlydneva. "O snizhenii skorosti svobodnyh fotonov pri modelirovanii ih rasprostraneniya v prostranstve S pomoshch'yu volnovoj funkcii $\mathrm{v}$ koordinatnom predstavlenii" [Electronic resource], in Trudy XIII mezhdunar. nauchno-tekhnicheskoj konf. APEHP - 2016, Tom 8, Novosibirsk, 2016, pp. 50-57. URL: https://cloud.mail.ru/public/FBMT/KugeZk8F7/TOM08.pdf (accessed 21 October 2017)

[47] A. P. Davydov, T. P. Zlydneva. "On the reduction of free photons speed in modeling of their propagation in space by the wave function in coordinate representation", in $201613^{\text {th }}$ International scientific-technical conference on actual problems of electronic instrument engineering (APEIE) - 39281 proceedings, Novosibirsk, vol. 1, part.2, pp. 233-240, 2016 DOI: 10.1109/APEIE.2016.7806458.

[48] D. Giovannini, J. Romero, V. Potoček, G. Ferenczi, F. Speirits, S. M. Barnett, D. Faccio, M.J. Padgett, "Spatially structured photons that travel in free space slower than the speed of light", Science, vol. 347 (6224), pp. 857-860, 2015.

[49] Z.L. Horváth, B. Major, "Comment on "Spatially structured photons that travel in free space slower than the speed of light", available at:: https://arxiv.org/ftp/arxiv/papers/1504/1504.06059.pdf (accessed 21 October 2017).

[50] G. Gouesbet, J.A. Lock, "On light traveling in free space slower than the speed of light and other curiosities associated to light propagation and light scattering", ELS-XV-2015, Abstracts, Leipzig, 2015, available at: http://meetingorganizer.copernicus.org/ELS-XV-2015/ELS-XV-201516.pdf (accessed 21 October 2017).

[51] R. Mignani, E. Recami, and M. Baido, "About a Diraclike Equation for the Photon, According to Ettore Majorana", Left. Nuovo Cimento, vol.11, № 12, pp. 568-572, 1974.

[52] A.P. Davydov, "O postroenii special'noj teorii otnositel'nosti (STO) iz simmetrii prostranstva i vremeni bez postulatov STO", Jelekrtromagnitnye volny i jelektronnye sistemy, vol. 8, № 1, pp. 49-58, 2003.

[53] A.P. Davydov, "Kurs lekcij po kvantovoj mehanike. Matematicheskij apparat kvantovoj mehaniki": Ucheb. posobie. Magnitogorsk: Izd-vo Magnitogorsk. gos. tehn. un-ta im. G.I. Nosova, 2014, 188 p.

[54] A.P. Davydov, "Foton kak kvazichastica pri vozbuzhdenii spinovoj volny v fizicheskom vakuume na plankovskih rasstojanijah", in Tezisy dokl. XLIV vnutrivuz. nauch. konf. Prepodav. MaGU "Sovremennye problem nauki i obrazovanija”. Magnitogorsk: Izd-vo Magnitogorsk. gos. un-ta, p. 174, 2006.

[55] A.P. Davydov, "Novye kvantovye ob\#ekty kosmomikrofiziki jelementarnye bessinguljarnye chernye dyry - kak sledstvie KJeD i OTO", in Sb. nauch. trudov "Fundametal'nye prikladnye issledovanija". Magnitogorsk: Izd-vo MGPI, pp. 22-41, 1997.

[56] A.P. Davydov, "Vozmozhnost' kvantovyh bessinguljarnyh chernyh dyr s plankovskimi parametrami i jekstremal'noj metrikoj $\mathrm{v}$ fizike $\mathrm{i}$ kosmologii”, Jelektromagnitnye volny i jelektronnye sistemy, vol. 3, № 2, pp. 67-78, 1998.

[57] A.P. Davydov, "Jekstremal'nye maksimony, struktura fundamental'nyh chastic, KJeD, OTO i RTG A.A. Logunova", Jelektromagnitnye volny i jelektronnye sistemy, vol. 6, № 5, pp. 4-13, 2001.

[58] J.F. Clauser, "Experimental distinction between the quantum and classical field theoretic predictions for the photo-electric effect", Phys. Rev. D, vol. 9, pp. 835-860,1974. 